\title{
Recollections of Parental Rejection, Self-Criticism and Depression in Suicidality
}

\author{
Rui C. Campos, Avi Besser, and Sidney J. Blatt
}

The present study examines whether self-criticism and depressive symptoms mediate the relationship between recollections of parental rejection and suicidality. A community sample of 200 Portuguese adults completed, in counterbalanced order, a socio-demographic questionnaire, the short form of the Inventory for Assessing Memories of Parental Rearing Behaviour (EMBU), the Depressive Experiences Questionnaire (DEQ), the Center for Epidemiologic Studies Depression Scale (CES-D), and reports of any suicide intention and/or ideation and suicide attempts. Structural Equation Modeling (SEM) indicated that recollections of parental rejection are significantly associated with depressive symptoms and suicidality. Recollections of parental rejection are indirectly associated with depressive symptoms and suicidality through self-criticism. The association between self-criticism and suicidality is mediated by depressive symptoms. In addition to a significant direct association between recollections of parental rejection and suicidality, the final model indicated that recollections of parental rejection are significantly associated with self-criticism. That same self-criticism is significantly associated with depressive symptoms which, in turn, are significantly associated with suicidality. Individuals with recollections of parental rejection are at greater risk for suicide ideation and behavior, possibly because such experiences predispose them to intense self-criticism which is a risk factor for depression associated with suicidal ideation and behavior.

Keywords depression, parental rejection, self-critical perfectionism, suicidality

\section{INTRODUCTION}

Suicide is a major mental health problem across the life span, but especially in adolescents and older adults. In Portugal, in 2009, 1025 persons died by suicide (WHO, 2012). The national official rate of suicide is 10.3 deaths per 100.000 people and suicide is the leading cause of unnatural death (National Institute of Statistics,
2011). There are several theoretical models for suicide and suicide behavior that include sociocultural and psychological perspectives (e.g., Goldney \& Schioldann, 2004), demonstrating the potential interaction among several risk factors (e.g., Beautrais, Collings, Ehrhardt et al., 2005). Early developmental vulnerabilities, like insecure attachment and malevolent object representations, as well as personality traits, 